\title{
CERTAIN METHOD FOR GENERATING A SERIES OF LOGICS
}

\author{
SATOSHI MIURA and SHÛRÔ NAGATA
}

Introduction. At first, we define three relations $\supseteq,=$, and $\supset$ in connection with a pair of logics $\boldsymbol{L}$ and $\boldsymbol{L}^{*}$ as follows:

$\boldsymbol{L} \supseteq \boldsymbol{L}^{*}$, if and only if every proposition provable in $\boldsymbol{L}^{*}$ is also provable in $\boldsymbol{L}$;

$\boldsymbol{L}=\boldsymbol{L}^{*}$, if and only if $\boldsymbol{L} \supseteq \boldsymbol{L}^{*}$ and $\boldsymbol{L}^{*} \supseteq \boldsymbol{L} ;$

$\boldsymbol{L} \supset \boldsymbol{L}^{*}$, if and only if $\boldsymbol{L} \supseteq \boldsymbol{L}^{*}$ but not $\boldsymbol{L}^{*} \supseteq \boldsymbol{L}$.

Next, for a logic $\boldsymbol{L}$, we denote by $\boldsymbol{L}[A]$ the fortified logic of $\boldsymbol{L}$ by regarding a proposition $A$ as a new axiom scheme.

By $\boldsymbol{L O Q}$, we denote the logic obtained by adjoining Peirce's rule,

$$
P \equiv((a \rightarrow b) \rightarrow a) \rightarrow a,
$$

to the primitive logic $\boldsymbol{L O}$ ( $c f$. Ono [6], [7]). According to Nagata [4], we can obtain a descending sequence, $\boldsymbol{L}_{1}, \boldsymbol{L}_{2}, \ldots$, from $\boldsymbol{L O Q}$ toward $\boldsymbol{L O}$ (i.e. $\left.\boldsymbol{L O Q}=\boldsymbol{L}_{1} \supset \boldsymbol{L}_{2} \supset \ldots \supset \boldsymbol{L}_{i} \supset \ldots \supset \boldsymbol{L O}\right)$ by the following method. A series of propositions $P_{i}$ is defined recursively as follows:

$$
\left\{\begin{array}{l}
P_{1} \equiv P, \\
P_{i+1} \equiv\left(\left(p_{i} \rightarrow P_{i}\right) \rightarrow p_{i}\right) \rightarrow p_{i}, \quad(i=1,2, \ldots),
\end{array}\right.
$$

where $p_{i}$ 's are mutually distinct proposition-variables not occurring in $P$. For the series $P_{1}, P_{2}, \ldots$, we can assert that

$$
\boldsymbol{L O Q}=\operatorname{LO}\left[P_{1}\right] \supset \operatorname{LO}\left[P_{2}\right] \supset \ldots \supset \operatorname{LO}\left[P_{i}\right] \supset \ldots \supset L O .
$$

We have noticed that, by making use of the same method, existence of descending sequences from $\boldsymbol{K}$-series logics (LQ, $\boldsymbol{L} \boldsymbol{N}, \boldsymbol{L K})$ toward their corresponding $\boldsymbol{J}$-series logics (LP, $\boldsymbol{L M}, \boldsymbol{L J}$ ) (cf. Ono [6]) can be proved. We have also noticed that existence of a descending sequence from $\boldsymbol{L} \boldsymbol{N}$ toward $\boldsymbol{L} \boldsymbol{D}=\boldsymbol{L} \boldsymbol{M}[a \bigvee \rightarrow a](c f$. Curry [1]) can be proved similarly.

Received September 6, 1966. 
Discussing with us our recent studies on the subject, Prof. T. Tugué pointed out that we would have, in a similar manner, a descending sequence toward a logic $\boldsymbol{L}$ by starting from any proposition $A$, not provable in $\boldsymbol{L}$, instead of starting from Peirce's rule. Guided by his valuable suggestion, we obtained the following conclusion.

For any proposition $A$, a series of propositions $A_{i}$ is defined recursively as follows :

$$
\left\{\begin{array}{l}
A_{1} \equiv A, \\
A_{i+1} \equiv\left(\left(p_{i} \rightarrow A_{i}\right) \rightarrow p_{i}\right) \rightarrow p_{i}, \quad(i=1,2, \ldots),
\end{array}\right.
$$

where $p_{i}$ 's are mutually distinct proposition-variables not occurring in $A$. The proposition $A$ is called kernel. Taking Peirce's rule $P$ as the kernel $A$, we can produce the descending sequences described before. If we take $a \vee \rightarrow a$ (law of the excluded middle) as the kernel $A$, we can produce a descending sequence from $\boldsymbol{L D}$ toward $\boldsymbol{L M}$. Along this line, we would be able to give other examples as many as we like. To show these facts, we shall use certain truth-table, called $(n, r)$-evaluation. The $(n, r)$-evaluation is a slight refining of the truth-table appearing in Gödel [2] ${ }^{1)}$. The refinement lies on the evaluation of negation defined as follows:

\begin{tabular}{c|ccccc}
$a$ & 0 & $1 \ldots$ & $\ldots-1$ & $r \ldots$ & $\ldots$ \\
\hline$\rightarrow a$ & $r$ & $r \ldots$ & $\ldots$ & $0 \ldots$
\end{tabular}

The main purpose of this paper is to show that, for a logic $\boldsymbol{L}$ and a kernel $A$, we can generate a descending sequence from $\boldsymbol{L}[A]$ toward $\boldsymbol{L}$ under certain conditions. We wish to express our thanks to Profs. K. Ono and T. Tugué for their kind guidances.

Definition. For integers $n$ and $r$ such that $1 \leq r \leq n$, any evaluation having the following truth-value properties is called $(n, r)$-evaluation:

$$
a \rightarrow b=\left\{\begin{array}{lll}
0 & \text { if } & a \geq b, \\
b & \text { if } & a<b,
\end{array}\right.
$$

1) In Gödel [2], it is discussed that there is "eine monoton abnehmende Folge von Systemen" between $\boldsymbol{L} \boldsymbol{K}$ and $\boldsymbol{L} \boldsymbol{J}$ by considering the formula $F_{n} \equiv \vee_{1 \leq i<k \leq n}\left(a_{i} \equiv a_{k}\right)$ with respect to a many-valued evaluation. This fact enables us to do the same discussion between $\boldsymbol{L K}$ and a logic, in which every provable proposition is $(n, r)$-true ( $c f$. Definition) for any $n$ and some $r$. Moreover, in Umezawa [8]-[10] and Nishimura [5], there are detailed discussions on intermediate logics between $\boldsymbol{L K}$ and $\boldsymbol{L} \boldsymbol{J}$. 


$$
\begin{aligned}
& a \vee b=\operatorname{Min}(a, b), \\
& a \wedge b=\operatorname{Max}(a, b), \\
& \neg a=\left\{\begin{array}{lll}
r & \text { if } a<r, \\
0 & \text { if } a \geq r ;
\end{array}\right.
\end{aligned}
$$

where the truth-values of propositions $a, b$, denoted simply by $a, b$, respectively, runs over the set $\{0,1, \ldots, n\}$. If we take the logical constant 人 (contradiction) whose truth-value is defined by $r$, and define $\rightarrow a$ by $a \rightarrow \wedge$, then the above truth-value property for negation is obtained. For the predicate logics, we take a domain of $k$ individual objects $\left\{\xi_{1}, \xi_{2}, \ldots, \xi_{k}\right\}$ and define the truth-value of

$$
\begin{aligned}
& (\xi) a(\xi) \quad \text { by } \quad a\left(\xi_{1}\right) \wedge a\left(\xi_{2}\right) \wedge \ldots \wedge a\left(\xi_{k}\right), \\
& (\exists \xi) a(\xi) \text { by } a\left(\xi_{1}\right) \vee a\left(\xi_{2}\right) \vee \ldots \vee a\left(\xi_{k}\right) .
\end{aligned}
$$

Any proposition whose truth-value is always 0 is called $(n, r)$-true.

For any $n$ and $r$, all the axiom schemes ${ }^{2)}$ of $\boldsymbol{L M}$ are $(n, r)$-true, and all the inference rules of $\boldsymbol{L M}$ deduce $(n, r)$-true conclusions, whenever their assumptions are all $(n, r)$-true. $\quad \wedge \rightarrow a$ is $(n, n)$-true, $a \vee \rightarrow a$ is $(n, 1)$-true, and (人 $\rightarrow a) \vee b \vee \rightarrow b$ ( $c f$. Example) is both $(n, 1)$ - and $(n, n)$-true for all $n, r$.

Before stating the theorem, the following two lemmas are remarkable.

Lemma 1. If the kernel $A \leq n-j(0 \leq j \leq n-1)$ for the $(n, r)$-evaluation, then, $A_{i} \leq n-j-i+1(1 \leq i \leq n-j+1)$.

Lemma 2. If the kernel $A$ takes the truth-value $n-j(0 \leq j \leq n-1)$ for the $(n, r)$-evaluation, then, $A_{i}$ takes $n-j-i+1(1 \leq i \leq n-j+1)$.

Theorem. Let $\boldsymbol{L}$ be a logic such that $\boldsymbol{L K} \supset \boldsymbol{L} \supseteq \boldsymbol{L O}$. Assume that there exists a function $r=r(n)(r=1,2, \ldots, n)$ satisfying the following conditions.

(1) For all $n$, every $\boldsymbol{L}$-provable ${ }^{3}$ proposition is $(n, r)$-true.

(2) There exists a non-negative integer $j$ such that, for all $n(n \geq 2)$ larger than $j$, a proposition $A$ can never takes the truth-value larger than $n-j$, but can take certainly $n-j$ by the $(n, r)$-evaluation.

Then, there is a descending sequence from $\boldsymbol{L}[A]$ toward $\boldsymbol{L}$, i.e.,

2) $c f . H$ system of Curry [1].

3) In this paper, for a $\operatorname{logic} \boldsymbol{L}$, a proposition $A$ is called to be $\boldsymbol{L}$-provable when $A$ is provable in $\boldsymbol{L}$. 


$$
\boldsymbol{L}[A]=\boldsymbol{L}\left[A_{1}\right] \supset \boldsymbol{L}\left[A_{2}\right] \supset \ldots \supset \boldsymbol{L}\left[A_{i}\right] \supset \ldots \supset \boldsymbol{L} .
$$

Proof. (i) The cases $j \neq 0$ or $n \geq 3$. If we take $(n+j-1, r)$ evaluation in place of $(n, r)$-evaluation, then, $A \leq n-j$ turns out to be $A \leq n-1$. By Lemma $1, A_{i} \leq n-i$ holds; hence, $A_{n}=0$ always holds. Since all the $\boldsymbol{L}$-provable propositions are $(n+j-1, r)$-true by assumption, all the $\boldsymbol{L}\left[A_{n}\right]$-provable propositions are always $(n+j-1, r)$-true. By Lemma 2, however, $A_{i}=n-i$ holds; hence, $A_{n-1}=1$ holds. Therefore, $A_{n-1}$ is not $\boldsymbol{L}\left[A_{n}\right]$-provable. Namely, $\boldsymbol{L}\left[A_{n-1}\right] \supset \boldsymbol{L}\left[A_{n}\right] \supset \boldsymbol{L}$.

(ii) The case $j=0$ and $n=2$. By assumption, there exists $r$ such that $A_{1}$ can take 2 by $(2, r)$-evaluation. However, $A_{2} \leq 1$ by Lemma 1 . Hence, $A_{1}$ is not $\boldsymbol{L}\left[A_{2}\right]$-provable. Therefore, $\boldsymbol{L}\left[A_{1}\right] \supset \boldsymbol{L}\left[A_{2}\right]$. q.e.d.

EXAmple. In the following table, descending sequences from $\boldsymbol{L}[A]$ toward $\boldsymbol{L}$ are exhibited by showing their kernels $A$ and the numbers $r$ appearing in the assumption of the theorem. We can further substitute, in the table, $\boldsymbol{L M}[\rightarrow a \vee \rightarrow \rightarrow a]$ or $\boldsymbol{L} \boldsymbol{M}[(a \rightarrow b) \vee(b \rightarrow a)]$ etc. for $\boldsymbol{L M}$. In the following table, $\boldsymbol{A} \cap \boldsymbol{B}$ denotes the logics in which any proposition is provable, if and only if it is both $\boldsymbol{A}$ - and $\boldsymbol{B}$-provable.

\begin{tabular}{|c|c|c|c|c|}
\hline & $\boldsymbol{L}[A]$ & $\boldsymbol{L}$ & $A$ & $r$ \\
\hline 1 & $L O Q$ & $\boldsymbol{L O}$ & $P \equiv((a \rightarrow b) \rightarrow a) \rightarrow a$ & $1 \leq r \leq n$ \\
\hline 2 & $\boldsymbol{L Q}$ & $\boldsymbol{L P}$ & $P$ & $1 \leq r \leq n$ \\
\hline 3 & $\boldsymbol{L} N$ & $\boldsymbol{L} \boldsymbol{M}$ & $P$ & $1 \leq r \leq n$ \\
\hline 4 & $\boldsymbol{L} \boldsymbol{K}$ & $\boldsymbol{L} \boldsymbol{J}$ & $P$ & $r=n$ \\
\hline 5 & $\boldsymbol{L} N$ & $\boldsymbol{L D}$ & $P$ & $r=1$ \\
\hline 6 & $\boldsymbol{L D}$ & $\boldsymbol{L} \boldsymbol{M}$ & $a \vee \rightarrow a$ & e.g. $r=n$ \\
\hline 7 & $\boldsymbol{L} \boldsymbol{J}$ & $\boldsymbol{L} \boldsymbol{M}$ & $\curlywedge \rightarrow a$ & $1 \leq r \leq n-1$ \\
\hline 8 & $\boldsymbol{L} \boldsymbol{N}_{i} \equiv \boldsymbol{L M}\left[P_{i}\right]$ & $\boldsymbol{L} \boldsymbol{D}_{i} \equiv \boldsymbol{L} \boldsymbol{M}\left[B_{i}\right](B \equiv a \bigvee \neg a)$ & $P_{i}$ & $r=1$ \\
\hline 9 & $\boldsymbol{L} \boldsymbol{N}_{i}$ & $\boldsymbol{L} \boldsymbol{J}_{i} \equiv \boldsymbol{L} \boldsymbol{M}\left[C_{i+1}\right](C \equiv \curlywedge \rightarrow a)$ & $P_{i}$ & $r=n$ \\
\hline 10 & $\boldsymbol{L} \boldsymbol{J} \cap \boldsymbol{L} \boldsymbol{N}$ & $L M$ & $(\Lambda \rightarrow a) \vee b \vee(b \rightarrow c)$ & $1 \leq r \leq n-1$ \\
\hline 11 & $L N$ & $\boldsymbol{L} \boldsymbol{J} \cap \boldsymbol{L} \boldsymbol{N}$ & $P$ & $r=n$ \\
\hline 12 & $\boldsymbol{L} \boldsymbol{J} \cap \boldsymbol{L} \boldsymbol{D}$ & $\boldsymbol{L} \boldsymbol{M}$ & $(\wedge \rightarrow a) \vee b \vee \rightarrow b$ & e. $g . r=n-1$ \\
\hline
\end{tabular}




\begin{tabular}{|c|c|c|c|c|}
\hline 13 & $\boldsymbol{L D}$ & $\boldsymbol{L} \cap \cap \boldsymbol{L D}$ & $a \vee \rightarrow a$ & $r=n$ \\
\hline 14 & $\boldsymbol{L} \boldsymbol{J}$ & $\boldsymbol{L} \boldsymbol{J} \cap \boldsymbol{L} \boldsymbol{D}$ & $\wedge \rightarrow a$ & $r=1$ \\
\hline 15 & $\boldsymbol{L} \boldsymbol{J} \cap \boldsymbol{L} \boldsymbol{N}$ & $\boldsymbol{L} \boldsymbol{J} \cap \boldsymbol{L} \boldsymbol{D}$ & $(人 \rightarrow a) \vee b \bigvee(b \rightarrow c)$ & $r=1$ \\
\hline
\end{tabular}

(As for the correlations of logics in the lines 10-15 under $\boldsymbol{L}[A]$ and $\boldsymbol{L}$, see Miura [3]. )

\section{REFERENCES}

[1] Curry, H.B., Foundations of mathematical logic (1963), New York.

[ 2 ] Gödel, K., Zum intuitionistischen Aussagenkalkül, Akad. Wiss. Anzeiger, vol. 69 (1932), 65-66.

[ 3 ] Miura, S., A remark on the intersection of two logics, Nagoya Math. J., vol. 26 (1966), 167-171.

[4] Nagata, S., A series of successive modifications of Peirce's rule, Proc. Japan Acad., vol. 42 (1966), 859-861.

[5] Nishimura, I., On formulas of one variable in intuitionistic propositional calculus, J. Symb. Logic, vol. 25 (1960), 327-331.

[6] Ono, K., On universal character of the primitive logic, Nagoya Math. J., vol. 27-1 (1966), 331-353.

[ 7 ] Ono, K., A certain kind of formal theories, Nagoya Math. J., vol. 25 (1965), 59-86.

[ 8 ] Umezawa, T., Über die zwischensysteme der Aussagenlogik, Nagoya Math. J., vol. 9 (1955), 181-189.

[9] Umezawa, T., On intermediate propositional logics, J. Symb. Logic, vol. 24 (1959), 20-36.

[10] Umezawa, T., On logics intermediate between intuitionistic and classical predicate logic, J. Symb. Logic, vol. 24 (1959), 141-153.

Toyota Technical College

and

Mathematical Institute,

Nagoya University

Added in proof April 28, 1967.

After this paper had been admitted, we found the fact that a series of propositions $P_{i}$, appearring in Nagata [4] and also in this paper, has been introduced by A.S. Troelstra in the slightly different form. (See [11] cited below.) A result of Nagata [4] has been already used in Troelstra [11] in order to verify one of his theorems. Moreover, there are some arguments in [11] connected with ours in the present paper.

[11] Troelstra, A.S., On intermediate propositional logics, Nederl. Akad. Wetensch. Proc. Ser. A, vol. 68 (Indag. Math., vol. 27) (1965), 141-152. 